\title{
Wheat Leaf Rust Detection at an Early Stage with Atomic Force Microscopy (AFM)
}

\author{
Zeeshan Haider ${ }^{1}$, Nouman Hassan ${ }^{2}$, Arshad Maryam², Shamaraz Firdous ${ }^{3 *}$, Uzma Aziz $^{3}$ and Arshad Mehmood $^{3}$ \\ ${ }^{1}$ Capital University of Science and Technology, Islamabad, Pakistan
}

${ }^{2}$ COMSATS Institute of Information Technology, Islamabad, Pakistan

${ }^{3}$ Biophotonics Laboratories, National Institute of lasers and Optronics (NILOP), Islamabad, Pakistan

*Corresponding author: Shamaraz Firdous, Biophotonics Laboratories, National Institute of lasers and Optronics (NILOP), Islamabad, Pakistan

\begin{abstract}
In this study, we demonstrated the early stage wheat rust diagnosis using Atomic Force Microscopy (AFM). Wheat rust is a common disease, caused by parasitic fungus and reduces crop yield up to 30 to 40 percent by effecting leaf and stem of plant. The wheat seed having fungus are the carrier of rust infection for the plant. The infected seed and rust fungus not only reduce food quantity but effect its quality. In our experimental research, the wheat leaf sample of day 10, 27, 35 and 45 were analyzed. The rust appears after day 24 on leaf, and rusted leaf has higher surface roughness than the normal one. We analyzed shape, surface structure of normal and rusted wheat leaf surface with AFM. The leaf protein and structure with high-resolution AFM imaging under controlled environmental conditions. The results revealed the morphological details of normal and viral infected proteins of wheat leaf with high-resolution in vivo study. The findings provide the basis for AFM as a useful tool for investigating microbial-surface structures and properties at an early stage of rust. We can visualize the starch granule surface at different stages of maturity reveals information regarding the development of granule architecture. The changes in infected leaf compared to normal can be seen at starch granule interior throughout the growth. Leaf surface shows depressions on the granule surface and changes in protein for the infected, and we can manage the disease at an early stage of rust development. These results will be used for an early stage detection of wheat rust and can be extended to other crops diseases detection using AFM and laser-scanning microscopy.
\end{abstract}

Keywords: Atomic Force Microscopy (AFM); Wheat Rust; Fungal Infection; viral detection

\section{Introduction}

In recent decades, there has been impressive growth in food production worldwide, which have been attributed to the development of improved, disease-resistant varieties, increased use of chemical fertilizers and pesticides. Although enough food is produced, but yet this food and the technology to produce it does not match the requirements and as a result about thousand million people do not get enough to eat and several millions die worldwide from hunger or hunger-related diseases [1-3]. During the period 1995 to 2050, the world's population is projected to increase by 75 percent and food security projected to become more critical, increasing wheat yield potential in the developing world remains a high priority [4]. Among the other fungal and viral infections, wheat leaf rust caused by viral fungus continue to pose a major threat to wheat production over large areas, particularly in Asia.
Rust diseases significantly influence several crop species and considerable research focuses on understanding the basis of host specificity and resistance. Like many pathogens, rust fungi vary considerably in the number of hosts they can infect, such as wheat leaf rust (Puccinia triticina), which can only infect species in the genera Triticum and Aegilops. Rusts often produce spots similar to leaf spots and are bright yellow, orange-red, reddish-brown or black in color. The pustules are usually raised above the leaf surface, and some types of rust occur on stems. Rusts are common on grains and grasses [5-7].

Wheat rusts spread rapidly over long distances by wind. If not detected and treated on time. For effective integrated management of wheat rust diseases close monitoring, international collaboration and strengthening of national capacities are crucial. Although in 
certain cases fungicide application may be necessary, preventive approaches are the most effective and environmentally friendly means of wheat rust management. specialists from international institutions and wheat producing countries work together to stop these diseases that involves continuous surveillance, sharing data and building emergency response plans to protect their farmers and those in neighboring countries. In general, a fungal infection can cause local or extensive necrosis and can inhibit normal growth of entire plant [8-10].

Several studies worldwide have been carried out for early rust detection. We have applied the surface morphological structure characterization using atomic force microscopy (AFM). It is a non-invasive method and are used for a variety of materials in surface science, biochemistry and biology [11-12]. It is a powerful technique and has the ability to obtain topographic information on and surface morphology of, the sample. It can also use to investigate chromosomes, proteins, living cells, carbohydrates and DNA [13]. In addition to obtain detailed structural information on the sample and allows us to visualize the cell surface properties on the nanometer scale [14]. Atomic force microscopy (AFM) provides images of biological structures without requiring labeling and to follow dynamic processes in real time. In structural biology, it has proven its ability to image proteins and protein conformational changes at sub molecular resolution, and in proteomics, it is developing as a tool to map surface proteomes and to study protein function by force spectroscopy methods. The power of AFM to combine studies of protein form and protein function enables bridging various research fields to come to a comprehensive, molecular level picture of biological processes [15]. In this study, a wheat leaf rust through the field experiment by the identification and disease index inversion is investigated successfully at an early stage with AFM. The aim of this study is to provide a method for monitoring and evaluating the diseases, so that proper management for rust protection can be made will in time.

\section{Materials and Methods}

The normal and rusted wheat leaf were collected during the season from National Agricultural Research Council (NARC). The samples were fixed, and changes are observed using the Atomic Force Microscopy (AFM, Alpha Contac, Germany). About 40 samples including 10 control and 30 rusted are taken from field. The AFM system installed at National Institute of lasers and Optronics
(NILOP) used to analyze the fresh sample taken from field in 1 to 2 hours. The sample of day 10,27, 35 and 45 are observed. The rust appears after day 24 on leaf, and rusted leaf has higher surface roughness than the normal one. The surface imaging was performed in the contact mode in air. Silicon nitride tips (Alpha Contac, Germany) were used for all AFM experiments. The radius of the cantilever of the tip was $7 \mathrm{~nm}$ and the diameter of the tip was 14 $\mathrm{nm}$. The length of the cantilever was $12.6 \mu \mathrm{m}$, thick-ness 3.52-4.08 $\mu \mathrm{m}$, width 30-31 $\mu \mathrm{m}$, and had an oscillation frequency of 287-336 $\mathrm{kHz}$ and a force constant of 28-45 N m-1. The images were analyzed by using WSXM 4.0 Develop 12.1 software for gaining information from the topography of the cells. The observation was performed inside a chamber at room temperature $[1,2,3,16]$.

\section{Results and Discussions}

The leaf rust, caused by Puccinia triticina, is an important disease in most wheat growing areas. The use of genetic resistance is the most economical and environmentally friendly way to combat this disease. For early stage rust fungus detection, several conventional methods are being utilized [17-18]. Atomic force microscopy is a powerful technique, which allows surface imaging of non- conducting samples in nanometer scale. In this experiment wheat leaf are imaged under ambient conditions, i.e. in air and with minimal sample preparations. The wheat corps of normal and rusted field are shown in Figure 1. Determining the time and scale of primary infections is also difficult with leaf rust because $P$. triticinainfected wheat crops show weak symptoms during the latent period of the disease. The samples were collected from the field for day 10, 27, 35 and 45 and shown in Figure 2. The rust appears after day 24 on leaf, and rusted leaf has higher surface roughness than the normal one Leaf rust symptoms are also checked several times to distinguish it by stem rust outbreaks at different time. Wheat leaf rust samples collection and examination of signs and symptoms in the field is very essential before AFM test. In some cases, diagnosis of leaf rust often requires isolation of the fungus and identification of the fungal pathotypes on differential host genotypes, which is complicated and time-consuming. Monitoring and early detection of this disease is crucial for the effective control and implementation of measures. Recent developments of remote sensing technology had the potential to enable direct detection of plant diseases under field conditions. However, sometime due to poor resolution the detection probability reduces.
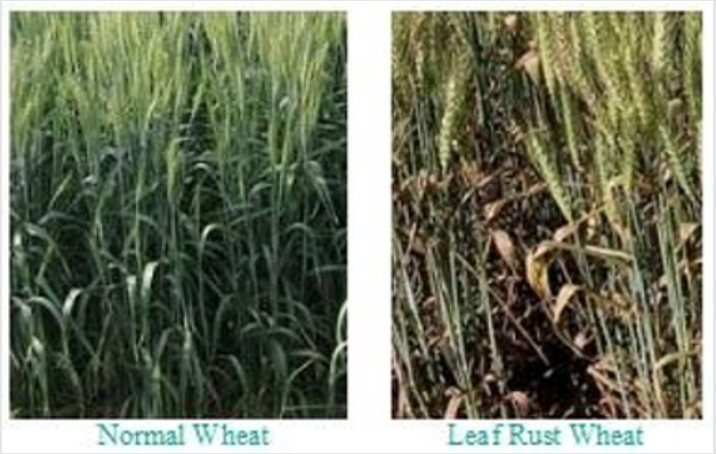

Figure 1: The field picture of wheat leaf normal and rust fungus infection crop overview. 

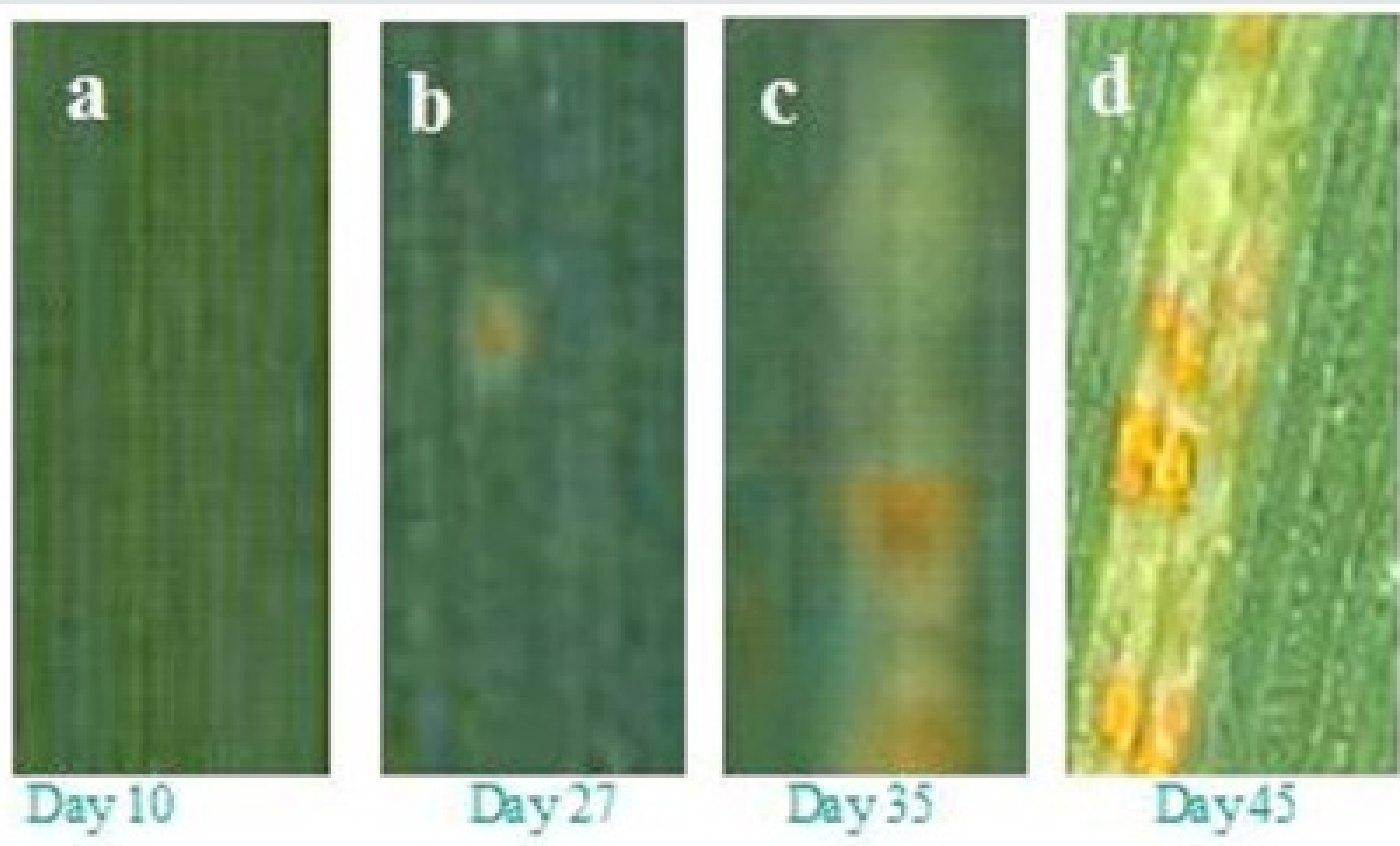

Day 27

Day 35

Day 45

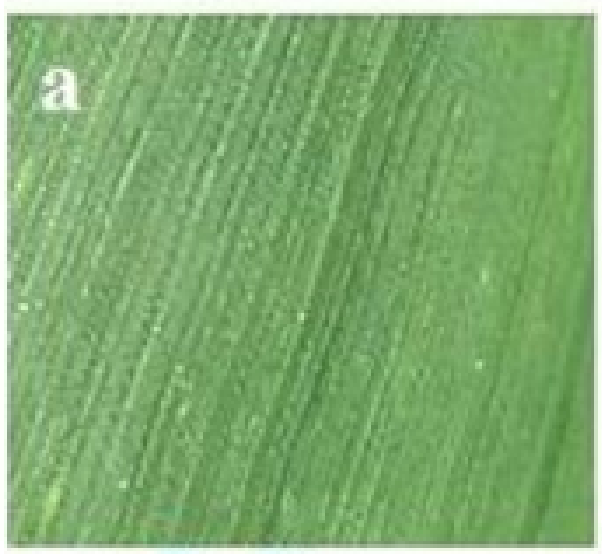

Day 10
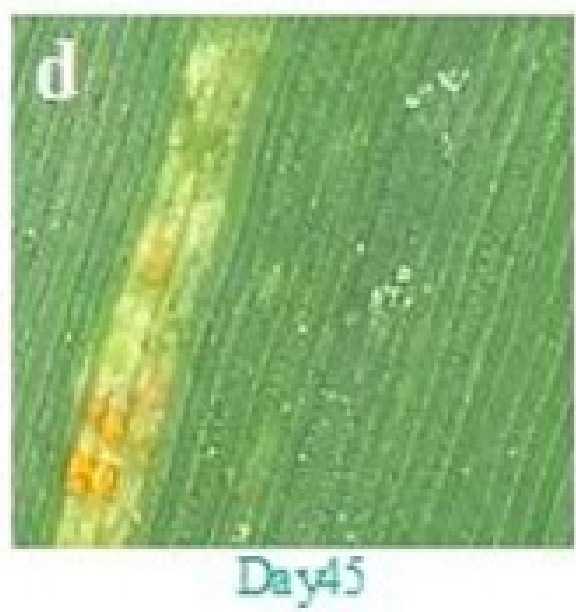

Figure 2: The wheat rusts fungal infected leaf samples of day 10 (a) normal and for day 27 (b), day 35(c) and day 45(d) are collected and analyzed with Atomic Force microscopy (AFM) topographic, phase images, and their cross-section analysis.

The AFM study of rusted wheat leaf from very early stage of growth provides a means to quantify their mechanical properties and examine their response to nanoscale forces, pulling single surface proteins with a functionalized tip allow one to understand their role in sensing and adhesion. The combination of these nanoscale techniques with modern molecular biology approaches, genetic engineering and optical microscopies provides a powerful platform for understanding the sophisticated functions of the plant machinery, and its role in the onset and progression of complex diseases. Topographic image and the cross-section analysis of the samples of day $10,27,35$ and 45 were imagine with AFM. The results can be seen in Figure 3 for the sample collected on day 10 (normal sample). It can be seen in Figures 2(a) \& 3, no fungus attack is observed on leaf and it represents normal study. The rust fungus appears after day 24 on leaf, and rusted leaf has higher surface roughness than the normal one at day 10. The leaf starch granule surface structure can be seen in Figure 3. The observation represents essentially a top view of the surface enabling estimations in two and three dimensions of the size of different microstructures. The rust fungus appears after day 24 and the sample in Figure 2(bd) and two and three-dimensional AFM images in Figure 4. As seen in the topographic image block lets are clearly visible in image. 

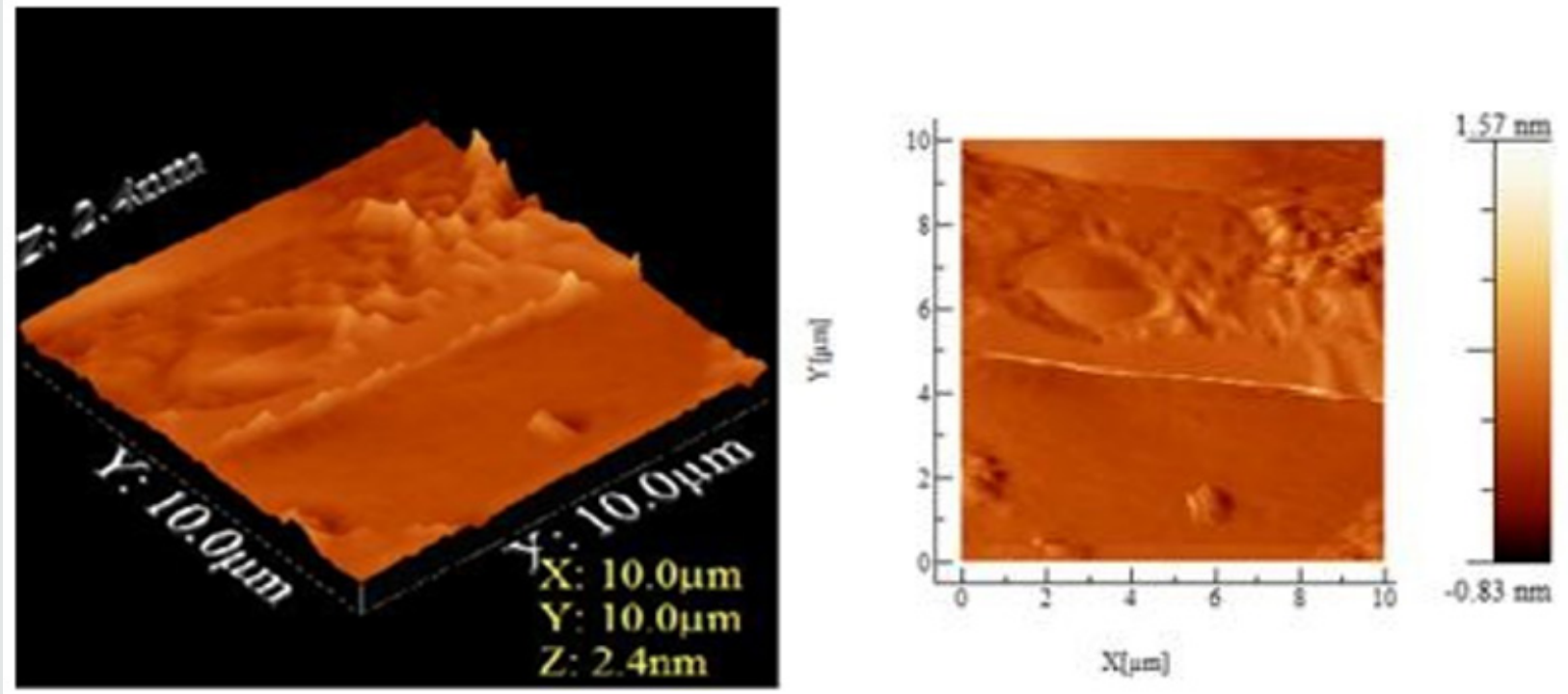

Figure 3: The Atomic Force microscopy (AFM) 2D and 3D topographic, phase image of Wheat leaf rust infection at day 10 for normal leaf. Scale of the axis of the cross-section analysis of topographic is $10 \mu \mathrm{m}$, angle 10-15o, whereas depth is $2.4 \mathrm{~nm}$.
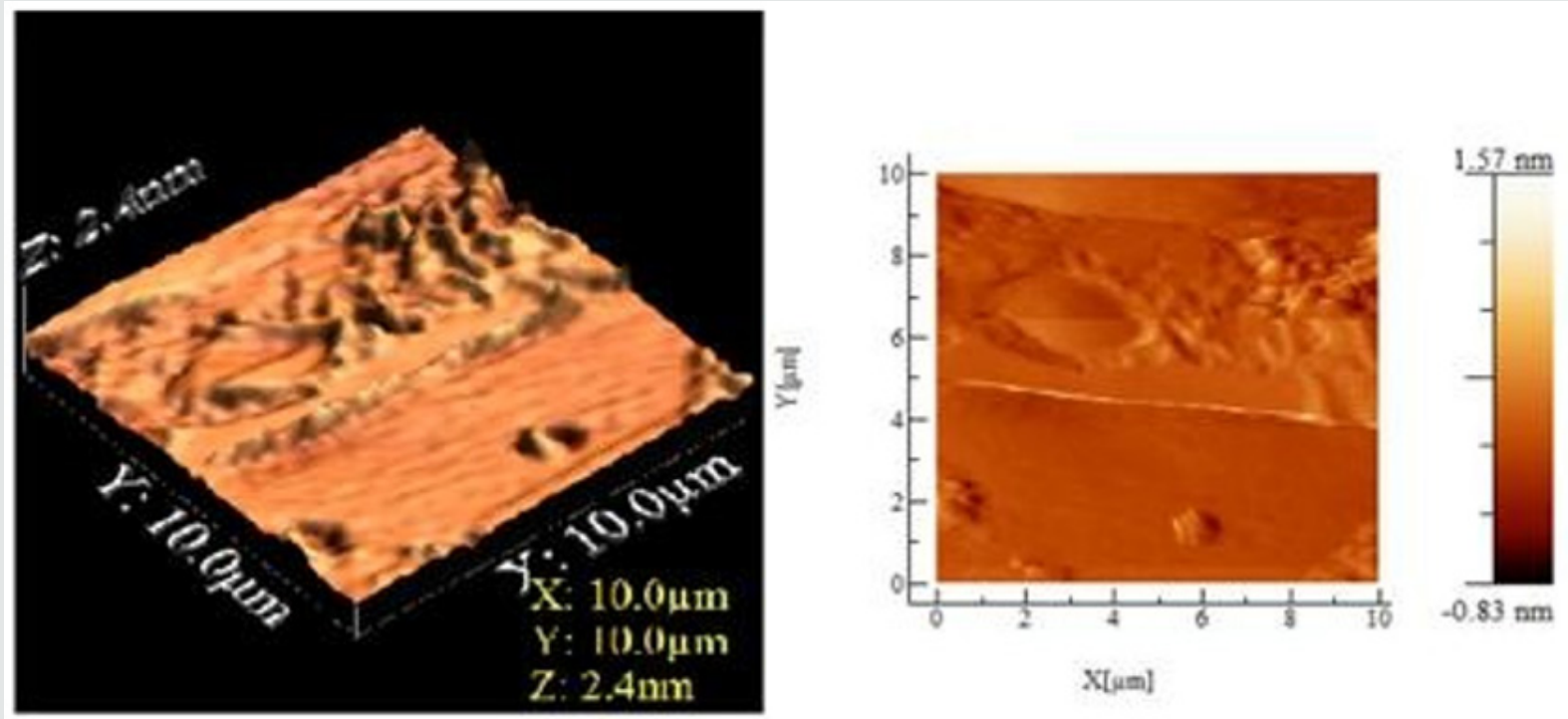

Figure 4: The Atomic Force microscopy (AFM) 2D and 3D topographic, phase image of Wheat leaf rust infection at day 45 for rust fungus infection. Scale of the axis of the cross-section analysis of topograph is $10 \mu \mathrm{m}$, angle 10-15o, whereas depth is 2.4 nm.

The AFM 2D and 3D images had dimensions between $10 \mathrm{x} 10$ $\mu \mathrm{m}$. Apparently, the blocklets were clustered or fused together at different heights forming nodules and are mostly elongated in shape. Depressions were also observed on the surface of the granule. Phase image in an AFM study highlights the stiffness of the sample surface. It records the phase lagging between the cantilever oscillation and the phase of driving signal giving an indication of relative stiffness at different locations of the surface of the sample, indicating rust formation. Thus, phase images can be utilized to recognize fungus and understand the microstructure inside depressions, which were hidden in the topographic image. The topographic image shows some corresponding features, surface roughness hinders the identification of domains. The phase image allows unambiguous resolution of the different material phases. The surface of the day 35 and 45 are similar to day 27 and indicate that top of the nodules was mostly stiffer than valleys, as shown in Figure 4.

The cross-section analysis of phase images, the stiffness of granule surfaces for normal and rusted leaf is different. The 
phase image further visualized the texture of surface depressions, which may be hidden in the topography, and the presence of deep gaps dividing bundles of nodules from each other. In contrast to the surface with blocklets commonly observed and reported in literature [19]. We can see that the presence of $4.5 \mathrm{~nm}$ deep depressions with higher stiffness similar to regular granule surface in the background of the amorphous surface suggests that these depressions likely extend to the underneath semi-crystalline growth ring. These depressions might also be a part of internal channels. The wheat tissue morphology can be described as a succession of more or less thick layers including the starchy endosperm layer, the protein stored as granules in the cells of plant seeds layer and the internal cells layers with external pericarp.

In past some researchers worked on an optical light detection for visible and near-infrared region to detect different types of rust at the leaf scale [20]. In this study, none of these indices were able to detect and discriminate the types of rust. However, the anthocyanin reflectance index can be used to detect yellow rust, and the transformed chlorophyll absorption and reflectance index can be used to detect leaf rust [21]. In another study conducted by Frank and Menz, hyperspectral and leaf multispectral data were used to estimate the severity of wheat leaf rust [22]. Results indicated that leaf rust could be detected in the early symptoms by using hyperspectral data. The algorithm used in this research was based on the minimum noise fraction transformation. The reflectance spectra of the infected, non-infected, and dry area, as well as the soil class were taken at the canopy level. Ashourloo et al. showed that the disease symptoms have a high impact on the infected plant reflectance spectra [23]. This means that as the disease severity increases, so does the collected spectrum variations at a specific disease severity. Results showed that as the disease severity increases, the scattering of the numerical values for all of the indices also increases. For the different amounts of scattering and classification accuracy are not the same and depend on the wavelength of light used. Wheat leaf rust at the leaf scale was studied for two purposes, one is to estimate the reflectance spectra of various disease symptoms; and other is to introduce an index for precise determination of disease severity using the spectral reflectance of leaf [24]. In our previous study we have applied optical detection techniques to several viral infection monitoring using human blood in vivo and in vitro [25-32].

\section{Conclusion}

In this, experimental studies we demonstrated that Atomic Force Microscopy AFM provides a powerful platform for detect wheat leaf rust fungus at nanoscale level. The main advantages of AFM are the ability to image and manipulate early stage fungus infection at nanometer resolution and its operation under a wide variety of physiological conditions for quantifying the physical properties of cellular structures and leaf surface molecules topography and structure morphology. The applied technique provides early stage detection of fungus pathogen, when it it cannot be observed visually. So, the protection management with anti-pathogen chemicals can be made to save the crop from disease. The development and combination of multiple orthogonal, yet complimentary, biophysical tools will clearly play a major role in illuminating a deeper understanding of the complex interplay between physical and biological information.

Collaborations between the Nano, physical and life sciences will lead to the AFM being used more routinely in studies of fundamental and complex biological processes. Such studies will lead to the understanding of the importance of the physical mechanisms governing fungal infection and its characteristics in biology. The atomic force microscopy (AFM) provides the structural, mechanical strength, topography, and surface morphology of the sample in easy way. The information related to the rust fungus infection at an early stage can facilitate the wheat management planning. AFM qualitative and quantitative imaging of granules and stomata support blocklet structural information of diverse starch systems. This imaging methodology will enhance other early stage rust detection techniques to be utilized for wheat food improvements.

\section{References}

1. Firdous S (2017) Optical Fluorescence Diagnostic of Wheat Leaf Rust with Laser Scanning Confocal Microscopy. Advances in Crop Sciences and Technology 6(2): 355 .

2. Carlile MJ, SC Watkinson, GW Gooday (2001) The Fungi (2 $2^{\text {nd }}$ edn) Academic Press, New York, USA pp. 608.

3. Hansen MA, RL Wick (1993) Plant disease diagnosis: present and future prospects. Advances in Plant Pathology 10: 65-126.

4. Horst R K (2001) Westcott's Plant Disease Handbook. ( $6^{\text {th }}$ edn) Kluwer Academic Publishers, Boston, MA, USA.

5. Kaminskyj SGW, Dahms TES (2008) High spatial resolution surface imaging and analysis of fungal cells using SEM and AFM. Micron 39(4): 349-361.

6. Anker CC, Niks RE (2001) Prehaustorial resistance to the wheat leaf rust fungus, Puccinia triticina, in Triticum monococcum (s.s.). Euphytica 117(3): 209-215.

7. Azinheira HG, Silva M, Talhinhas P, Medeira C, Maia I, et al. (2010) Nonhost resistance responses of Arabidopsis thaliana to the coffee leaf rust fungus (Hemileia vastatrix). Botany 88: 621-629.

8. Agrios G N (2005) Plant Pathology. Elsevier Academic Press Burlington, MA, USA pp. 952.

9. Bolton MD, Kolmer JA, Garvin DF (2008) Wheat leaf rust caused by Puccinia triticina. Molecular Plant Pathology 9(5): 563-576.

10. Bernoux M, Ve T, Williams S, Warren C, Hatters D, et al. (2011) Structural and functional analysis of a plant resistance protein TIR domain reveals interfaces for self- association, signaling, and autoregulation. Cell Host Microbe 9(3): 200-211.

11. Kirby AR (2011) Atomic force microscopy of plant cell walls. Methods Mol Biol 715:78-169.

12. Serfling A, Templer SE, Winter P, Ordon F (2016) Microscopic and Molecular Characterization of the Prehaustorial Resistance against Wheat Leaf Rust (Puccinia triticina) in Einkorn (Triticum monococcum). Front Plant Sci 9(7): 1668.

13. Carey MF, Peterson CL, Smale ST (2012) Experimental strategies for the identification of DNA- binding proteins. Cold Spring Harbor Protocols 1: 18-33.

14. Canet, D, Rohr R, Chamel A, Guillain F (1996) Atomic force microscopy study of isolated ivy leaf cuticles observed directly and after embedding in Epon. New Phytol 134(4): 571-577. 
15. Eaton P, West P (2010) Atomic force microscopy. Oxford: Oxford University Press, UK, pp. 116-117.

16. Binnig G, Quate CF, Gerber C (1986) Atomic force microscope. Physical Review Letters 56 (9): 930-933.

17. Perkins MC, Roberts CJ, Briggs D, Davies MC, Friedman A, et al. (2005) Surface morphology and chemistry of Prunus laurocerasus L. leaves: a study using X-ray photoelectron spectroscopy, time-of-flight secondaryion mass spectrometry, atomic-force microscopy and scanning electron microscopy. Planta. 221(1): 123-134.

18. Koch K, Neinhuis C, Ensikat HJ, Barthlott, W (2004) Self-assembly of epicuticular waxes on living plant surfaces imaged by atomic force microscopy (AFM). J Exp Bot 55(397): 711-718.

19. Zhao L, Schaefer D, Marten MR (2005) Assessment of elasticity and topography of Aspergillus nidulans spores via atomic force microscopy. Appl Environ Microbiol 71(2): 955-960.

20. Devadas R, Lamb DW, Simpfendorfer S, Backhouse D (2009) Evaluating ten spectral vegetation indices for identifying rust infection in individual wheat leaves. Precis. Agric 10: 459-470.

21. Griffiths PR (2009) Infrared and Raman instrumentation for mapping and imaging. In Infrared and Raman spectroscopic imaging, Edited by: Salzer R, Siesler pp. 656.

22. Franke J, Menz G (2007) Multi-temporal wheat disease detection by multi-spectral remote sensing. Precis. Agric 8(3): 161-172.

23. Ashourloo D, Mobasheri MR, Huete A (2014) Evaluating the effect of different wheat rust disease symptoms on vegetation indices using hyperspectral measurements. Remote Sens 6(6): 5107-5123.

24. Micic M, Hu D, Suh YD, Newton G, Romine M, et al. (2004) Correlated atomic force microscopy and fluorescence lifetime imaging of live bacterial cells. Colloids Surf. B: Biointerfaces 34(4): 205-212.
25. Saira Tariq, Muhammad Bilal, Shaheen Shahzad, Shamaraz Firdous, Uzma Aziz, et al. (2017) Diagnosis of thalassemia and iron deficiency anemia using confocal and atomic force microscopy. Laser Physics Letters, 14(11): 115703.

26. S Anwar, S Firdous (2016) Optical diagnosis of dengue virus infected human blood using Mueller matrix polarimetry. Optics and Spectroscopy 121 (2): 322-325.

27. Shamaraz Firdous, Shahzad Anwar (2016) Noninvasive optical diagnostic of breast cancer using depolarization of light, International Journal for Light and Electron Optics. Optik 127(5): 3035-3038.

28.S Anwar, S Firdous (2015) Optical diagnostic of Hepatitis B(HBV) and C (HCV) from Human blood serum using Raman Spectroscopy. Laser Physics Letter 12(7): 076001.

29. Shamaraz Firdous, Shahzad Anwar (2015) Dengue viral infection monitoring from diagnostic to recovery using Raman spectroscopy. Laser Physics Letter 12(8): 085601.

30. S Anwar, S Firdous, A Rehman, M Nawaz (2015) Raman Optical Diagnostic of Breast Cancer using Raman, Polarimetric Fluorescence Spectroscopy. Laser physics Letter 12(4): 045601.

31.S Firdous, M Nawaz, M Ahmed, S Anwar, A Rehman, et al. (2012) Measurement of Diabetic Sugar Concentration in Human Blood Using Raman Spectroscopy. Laser Physics 22 (6): 1090-1094.

32.S Firdous, M Ahmed, A Rehman, M Nawaz, S Anwar, et al. (2012) Transmission Spectroscopy of Dengue Viral Infection. Laser Physics Latter 9(4): 317-321.

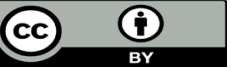

This work is licensed under Creative Commons Attribution 4.0 License

To Submit Your Article Click Here: Submit Article

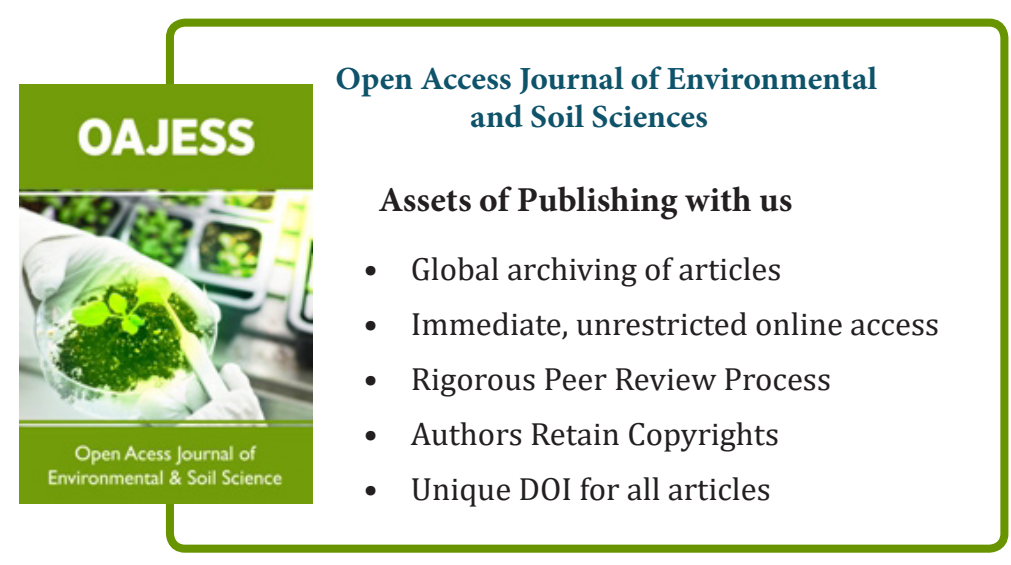

